DEMONSTRATIO MATHEMATICA

Vol. XLVII No $2 \quad 2014$

\title{
Hernando Gaitán
}

\section{ENDOMORPHISMS OF IMPLICATION ALGEBRAS}

\begin{abstract}
In this note we prove that if two implication algebras have isomorphic monoids of endomorphisms then they are isomorphic.
\end{abstract}

It is known that Boolean algebras (they are the algebraic counterpart of classical propositional logic) are determined by their endomorphism monoids (see [4, 5, 7]). Implication algebras, also called Tarski algebras or semi-Boolean algebras (see [1, 6]), are the algebraic counterpart of the implication fragment of classical propositional logic. Implication algebras form a variety which corresponds to the $\{\rightarrow\}$-subreducts of Boolean algebras. For a given implication algebra $\mathbf{A}$, we denote the monoid of its endomorphisms by $\operatorname{End}(\mathbf{A})$. In [3], we prove that finite implication algebras are determined by their endomorphisms. In this paper, we remove the finiteness hypothesis proving the following result:

Theorem 1. Let $\mathbf{A}_{1}$ and $\mathbf{A}_{2}$ be two implication algebras. Then

$$
\mathbf{A}_{1} \cong \mathbf{A}_{2} \text { iff } \operatorname{End}\left(\mathbf{A}_{1}\right) \cong \operatorname{End}\left(\mathbf{A}_{2}\right) \text {. }
$$

In [2] Celani and Cabrer prove that Tarski spaces with $T$-partial functions as arrows (see definitions below) form a category and this category is dually equivalent to the algebraic category of implication algebras.

A Tarski space (T-space for short) is a structure $\mathcal{X}=\langle X, \mathcal{K}, \tau\rangle$ such that

(1) $\langle X, \tau\rangle$ is a topological space where $\mathcal{K}$ is a basis for $\tau$ consisting of compact open subsets.

(2) If $A, B \in \mathcal{K}$ then $A \cap B^{\complement} \in \mathcal{K}$.

(3) If $x, y \in X$ and $x \neq y$ then there exists $U \in \mathcal{K}$ such that $x \notin U$ and $y \in U$.

2010 Mathematics Subject Classification: 06D30.

Key words and phrases: implication algebra, Tarski algebra, monoid of endomorphisms.

(C) Copyright by Faculty of Mathematics and Information Science, Warsaw University of Technology 
(4) If $F$ is a closed subset and $\left\{U_{i}: i \in I\right\}$ is a directed subfamily of $\mathcal{K}$ such that $F \cap U_{i} \neq \emptyset$ for all $i \in I$ then $F \cap\left(\bigcap\left\{U_{i}: i \in I\right\}\right) \neq \emptyset$.

It follows from conditions (1), (2) and (3) that a $T$-space is a Hausdorff space and consequently, every compact open set is closed (and hence, clopen). Also, it follows from (2) that $\emptyset \in \mathcal{K}$.

A $T$-partial function between two $T$-spaces $\mathcal{X}_{1}$ and $\mathcal{X}_{2}$ is a partial map $\varphi: X_{1} \longrightarrow X_{2}$ such that $\varphi^{-1}(U) \in \mathcal{K}_{1}$ for all $U \in \mathcal{K}_{2}$.

If $\mathcal{X}$ is a $T$-space, a $T$-partial function between $\mathcal{X}$ and itself is called a $T$-partial endomorphism of $\mathcal{X}$. We denote the monoid of $T$-partial endomorphisms of $\mathcal{X}$ by $\operatorname{Pend}(\mathcal{X})$.

In the light of the equivalence of Celani and Cabrer (see details of this equivalence in [2]), Theorem 1 is equivalent to the following theorem:

Theorem 2. Let $\mathcal{X}_{1}$ and $\mathcal{X}_{2}$ be two Tarski spaces. Then

$$
\mathcal{X}_{1} \cong \mathcal{X}_{2} \text { iff } \operatorname{Pend}\left(\mathcal{X}_{1}\right) \cong \operatorname{Pend}\left(\mathcal{X}_{2}\right) .
$$

Here, $\mathcal{X}_{1} \cong \mathcal{X}_{2}$ means that there exists a $T$-function from $X_{1}$ onto $X_{2}$ (i.e., a $T$-partial function with domain $X_{1}$ ) which is a bijection and such that its inverse is also a $T$-function (see Lemma 4.8 in [2]).

So, instead of proving Theorem 1, we prove Theorem 2. With this purpose, we introduce next an important family of $T$-partial functions: Let $\mathcal{X}:=\langle X, \mathcal{K}, \tau\rangle$ be a Tarski space. For each $U \in \mathcal{K}$ and $a \in X$, define the partial function $\varphi_{U, a}$ on $\mathrm{X}$ with domain $U$ by the rule $\varphi_{U, a}(x)=a$ if $x \in U$. Clearly, $\varphi_{U, a}$ is a $T$-partial endomorphism because for $V \in \mathcal{K}$,

$$
\varphi_{U, a}^{-1}(V):= \begin{cases}U & \text { if } a \in V, \\ \emptyset & \text { otherwise. }\end{cases}
$$

We now state and prove various propositions and lemmas from which our main result will follow easily. Our first proposition is an easy consequence of the definition of $\varphi_{U, a}$.

Proposition 1. For any $T$-partial endomorphism $\psi$, any $a \in X$ and any $U \in \mathcal{K}$, the following hold:

(i) $\varphi_{\emptyset, a}=\emptyset$.

(ii) $\psi \circ \varphi_{U, a}= \begin{cases}\varphi_{U, \psi(a)} & \text { if } a \text { is in the domain of } \psi, \\ \emptyset & \text { otherwise. }\end{cases}$

(iii) $\varphi_{U, a} \circ \psi=\varphi_{\psi^{-1}(U), a}$. 
In what follows, $\mathcal{X}_{i}:=\left\langle X_{i}, \mathcal{K}_{i}, \tau_{i}\right\rangle(i=1,2)$ will denote a Tarski space and $\Phi: \operatorname{Pend}\left(\mathcal{X}_{1}\right) \longrightarrow \operatorname{Pend}\left(\mathcal{X}_{2}\right)$, a monoid isomorphism. The next lemma asserts that the $T$-partial endomorphisms $\varphi_{U, a}$ are preserved under monoid isomorphisms.

\section{LEMMA 1.}

(i) $\Phi(\emptyset)=\emptyset$.

(ii) For $U_{1} \in \mathcal{K}_{1} \backslash\{\emptyset\}$ and $a_{1} \in X_{1}$ there exist $U_{2} \in \mathcal{K}_{2}$ and $a_{2} \in X_{2}$ such that $\Phi\left(\varphi_{U_{1}, a_{1}}\right)=\varphi_{U_{2}, a_{2}}$.

Proof. (i) It follows from the following facts: in any Tarski space, $\emptyset=\psi \circ \emptyset=$ $\emptyset \circ \psi$ for each partial endomorphism $\psi$ and any element in a semigroup with such a property is unique.

(ii) Set $\Psi:=\Phi^{-1}$ and $\sigma:=\Phi\left(\varphi_{U_{1}, a_{1}}\right)$. By injectivity of $\Phi, \Phi\left(\varphi_{U_{1}, a_{1}}\right) \neq \emptyset$. Let $b \in \operatorname{Im} \sigma$ and $c \in X_{2}$ such that $\sigma(c)=b$. Pick $U \in \mathcal{K}_{2}$ such that $b \in U$. By Proposition 1 (iii), $\varphi_{U, b} \circ \sigma=\varphi_{\sigma^{-1}(U), b}$. Observe that $\emptyset \neq \sigma^{-1}(U) \in \mathcal{K}_{2}$ and consequently, $\varphi_{\sigma^{-1}(U), b} \neq \emptyset$. Then, on one hand we have

$$
\Psi\left(\varphi_{U, b}\right) \circ \varphi_{U_{1}, a_{1}}=\Psi\left(\varphi_{U, b}\right) \circ \Psi(\sigma)=\Psi\left(\varphi_{\sigma^{-1}(U), b}\right)
$$

and, on the other hand, using Proposition 1 (ii) we have

$$
\Psi\left(\varphi_{U, b}\right) \circ \varphi_{U_{1}, a_{1}}=\varphi_{U_{1}, d}
$$

where $d=\Psi\left(\varphi_{U, b}\right)\left(a_{1}\right)$ and, as a byproduct, $a_{1}$ is in the domain of $\Psi\left(\varphi_{U, b}\right)$. So,

$$
\Phi\left(\varphi_{U_{1}, d}\right)=\varphi_{\sigma^{-1}(U), b} .
$$

Now pick $V \in \mathcal{K}_{1}$ such that $d \in V$. Clearly, for $t \in U_{1}$ we have

$$
\varphi_{V, a_{1}} \circ \varphi_{U_{1}, d}(t)=\varphi_{U_{1}, a_{1}}(d)=a_{1},
$$

so, $\varphi_{V, a_{1}} \circ \varphi_{U_{1}, d}=\varphi_{U_{1}, a_{1}}$ and, by applying $\Phi$ on both sides of this equality, we get $\Phi\left(\varphi_{U_{1}, a_{1}}\right)=\Phi\left(\varphi_{V, a_{1}}\right) \circ \varphi_{\sigma^{-1}(U), b}$ so that, for $t \in \sigma^{-1}(U)$ we have $\Phi\left(\varphi_{U_{1}, a_{1}}\right)(t)=\Phi\left(\varphi_{V, a_{1}}\right)(b)$ which means that $\Phi\left(\varphi_{U_{1}, a_{1}}\right)=\varphi_{\sigma^{-1}(U), e}$ where $e=\Phi\left(\varphi_{V, a_{1}}\right)(b)$.

Lemma 2. $\Phi\left(\varphi_{U, a}\right)=\varphi_{V, b}$ and $\Phi\left(\varphi_{U, a^{\prime}}\right)=\varphi_{V^{\prime}, b^{\prime}}$ imply $V=V^{\prime}$.

Proof. Pick $W \in \mathcal{K}_{1}$ such that $a^{\prime} \in W$. Then, for $t \in U, \varphi_{W, a} \circ \varphi_{U, a^{\prime}}(t)=$ $\varphi_{W, a}\left(a^{\prime}\right)=a$; so $\varphi_{W, a} \circ \varphi_{U, a^{\prime}}=\varphi_{U, a}$ and, by taking the images under $\Phi$ on both sides of this equality we get $\Phi\left(\varphi_{W, a}\right) \circ \varphi_{V^{\prime}, b^{\prime}}=\varphi_{V, b}$. From another side, for $t \in V^{\prime}, \Phi\left(\varphi_{W, a}\right) \circ \varphi_{V^{\prime}, b^{\prime}}(t)=\Phi\left(\varphi_{W, a}\right)\left(b^{\prime}\right)$, that is, $\Phi\left(\varphi_{W, a}\right) \circ \varphi_{V^{\prime}, b^{\prime}}=\varphi_{V^{\prime}, c}$, where $c=\Phi\left(\varphi_{W, a}\right)\left(b^{\prime}\right)$. So, we have shown that $\varphi_{V, b}=\varphi_{V^{\prime}, c}$ and this means that $V=V^{\prime}($ and $b=c)$.

Lemma 3. If $U \neq \emptyset \neq U^{\prime}$ then, $\Phi\left(\varphi_{U, a}\right)=\varphi_{V, b}$ and $\Phi\left(\varphi_{U^{\prime}, a}\right)=\varphi_{V^{\prime}, b^{\prime}}$ imply $b=b^{\prime}$. 
Proof. Pick $W \in \mathcal{K}_{1}$ such that $a \in W$ and, by virtue of Lemma 1, we may set $\Phi\left(\varphi_{W, a}\right)=\varphi_{W^{\prime}, c}$ for some $W^{\prime} \in \mathcal{K}_{2}$ and $c \in X_{2}$. Then, as $\varphi_{W, a} \circ \varphi_{U, a}=\varphi_{U, a}$, by taking the images under $\Phi$ on both sides of this equality, we get $\varphi_{W^{\prime}, c} \circ$ $\varphi_{V, b}=\varphi_{V, b}$. But for $t \in V$ we have $\varphi_{W^{\prime}, c} \circ \varphi_{V, b}(t)=\varphi_{W^{\prime}, c}(b)=\varphi_{V, b}(t)=b$ from which it follows that $c=b$; so, $\Phi\left(\varphi_{W, a}\right)=\varphi_{W^{\prime}, b}$. With the same argument but applied now to $\varphi_{U^{\prime}, a}$ we conclude that $\varphi_{W^{\prime}, b} \circ \varphi_{V^{\prime}, b^{\prime}}=\varphi_{V^{\prime}, b^{\prime}}$. Since for $t \in V^{\prime}$ we have that $\varphi_{V^{\prime}, b^{\prime}}(t)=b^{\prime}$ then $b^{\prime}=\varphi_{W^{\prime}, b} \circ \varphi_{V^{\prime}, b^{\prime}}(t)=$ $\varphi_{W^{\prime}, b}\left(b^{\prime}\right)=b$ (and, as a byproduct. $\left.b^{\prime} \in W^{\prime}\right)$.

Define $\phi: X_{1} \longrightarrow X_{2}$ by the rule $\phi(x)=y$ iff for every $V \in \mathcal{K}_{2}$, there exists $U \in \mathcal{K}_{1}$ such that $\Phi\left(\varphi_{U, x}\right)=\varphi_{V, y}$. By Lemma 3, $\phi$ is well defined and by Lemma 2, $\phi$ is one to one. Indeed, if $\Psi:=\Phi^{-1}$ and we define $\psi: X_{2} \longrightarrow X_{1}$ by the rule

$\psi(y)=x$ iff for every $U \in \mathcal{K}_{1}$, there exists $V \in \mathcal{K}_{2}$ such that

$$
\Psi\left(\varphi_{V, y}\right)=\varphi_{U, x} .
$$

It is easy to check that $\psi=\phi^{-1}$ so that $\phi$ is a bijection.

Proposition 2. $\phi$ and $\phi^{-1}$ are T-functions.

Proof. Since $\phi$ and $\phi^{-1}$ are defined symmetrically using $\Phi$, it is enough to prove the result just for $\phi$. Let $V \in \mathcal{K}_{2}$ and $x \in \phi^{-1}(V)$. Let $U \in \mathcal{K}_{1}$ such that

$$
\Phi\left(\varphi_{U, x}\right)=\varphi_{V, \phi(x)} .
$$

Since $\phi(x) \in V, \varphi_{V, \phi(x)} \circ \varphi_{V, \phi(x)}=\varphi_{V, \phi(x)}$. It follows from this that $x \in U$ (if $x \notin U$ then $\varphi_{U, x} \circ \varphi_{U, x}=\emptyset$ and by taking the images under $\Phi$ we have $\varphi_{V, \phi(x)} \circ \varphi_{V, \phi(x)}=\emptyset$, a contradiction) and this proves that $\phi^{-1}(V) \subseteq U$. Conversely, suppose that $x \in U$. Then, again from (1) it follows that $\phi(x) \in$ $V$. So, $\phi^{-1}(V)=U$ and this prove that $\phi$ is a T-function.

Conclusion: Theorem 2 follows at once from the previous results and Theorem 1 follows from the equivalence of Celani and Cabrer.

\section{References}

[1] J. C. Abbott, Semi-Boolean algebras, Mat. Vesnik 19 (1967), 177-198.

[2] S. A. Celani, L. M. Cabrer, Topological duality for Tarski algebras, Algebra Universalis 58 (2008), 73-94.

[3] H. Gaitán, Finite Tarski algebras are determined by their endomorphisms, Semigroup Forum 84 (2012), 25-32.

[4] C. J. Maxon, On semigroups of Boolean ring endomorphism, Semigroup Forum 4 (1972), 78-82. 
[5] K. D. Magill, The semigroup of endomorphisms of a Boolean ring, J. Aust. Math. Soc. 11 (1970), 411-416.

[6] H. Rasiowa, An Algebraic Approach to Non-Classical Logics, North Holland, Studies in Logic and the Foundations of Mathematics, Vol 78, Amsterdam, 1974.

[7] B. M. Schein, Ordered sets, semilattices, distributive lattices and Boolean algebras with homomorphic endomorphism semigroups, Fund. Math. 68 (1970), 31-50.

H. Gaitán

DEPARTAMENTO DE MATEMÁTICAS

FACULTAD DE CIENCIAS

UNIVERSIDAD NACIONAL DE COLOMBIA

CIUDAD UNIVERSITARIA

BOGOTÁ, COLOMBIA

E-mail: hgaitano@unal.edu.co

Received September 25, 2012; revised version March 25, 2013.

Communicated by A. Romanowska. 